\title{
Portal vein tumor thrombus in advanced hepatocellular carcinoma: A case report
}

\author{
XIANLI CHEN, LIGUAN LIU and XINGNAN PAN \\ Institute for Infectious Diseases, The 180th Hospital of The People's Liberation Army, Quanzhou, Fujian 362000, P.R. China
}

Received June 21, 2014; Accepted March 2, 2015

DOI: $10.3892 / \mathrm{ol} .2015 .3115$

\begin{abstract}
Portal vein tumor thrombus (PVTT) in hepatocellular carcinoma (HCC) is a common entity. However, the prognosis of patients with hepatocellular carcinoma (HCC) with portal vein tumor thrombus (PVTT) is extremely poor. The current study reports a case of HCC with PVTT, including a description of the gross surgical pathology and discussion of the relevance of assessing the growth of the tumor thrombus to the outcome of this disease. This case suggests that destruction of the primary tumor and PVTT must be considered according to the growth characteristics and modality of PVTT. It also indicates that transcatheter arterial embolization may be a suitable strategy for palliative treatment of patients with advanced HCC with PVTT.
\end{abstract}

\section{Introduction}

Hepatocellular carcinoma (HCC) is the sixth most common type of cancer and the third highest cause of cancer-related mortality worldwide (1). It is particularly prevalent in China, where it is the most common cause of cancer-related mortalities among patients with chronic Hepatitis B infection and cirrhosis (2). Globally, $>500,000$ new cases are diagnosed every year and the annual incidence rate is $2.5-7 \%$ in patients with liver cirrhosis (1). HCC exhibits intense neo-angiogenic activity during its progression. In the early stages the tumor is not highly vascularised and its blood supply comes from the portal vein. However, as the tumor grows the blood supply becomes progressively arterialized (3).

Correspondence to: Professor Xingnan Pan, Institute for Infectious Diseases, The 180th Hospital of The People's Liberation Army, 180 Huayuan Road, Quanzhou, Fujian 362000, P.R. China E-mail: cx17296@126.com

Abbreviations: HCC, hepatocellular carcinoma; PVTT, portal vein tumor thrombus; AFP, alpha-fetoprotein; OLT, orthotopic liver transplantation; TAE, transcatheter arterial embolization

Key words: Portal vein tumor thrombus, hepatocellular carcinoma
HCC has a tendency to invade the portal venous system, which results in portal vein tumor thrombus (PVTT); this is typically observed in the branches and trunk of the portal vein in $40-90 \%$ of patients whose HCC is advanced at the time of initial diagnosis (4). PVTT is an independent predictor for poor overall survival rate (5). The survival time after diagnosis of PVTT has been reported to be $<3$ months without treatment (6). These patients have extremely poor prognoses and a high rate of mortality. Previous studies have reported the median survival period of patients with portal venous invasion to be 2.7-4.0 months if left untreated (7).

Although many advances have been made in the diagnosis and treatment of HCC with PVTT, the prognosis remains poor. The survival of patients with this condition is dependent upon the PVTT growth characteristics, including the location and extent of portal vein involvement. PVTT may cause the interruption or reduction of the portal vein blood flow, which results in worsening of acute liver failure. Concomitantly, PVTT may increase the portal vein blood pressure, leading to the aggravation of gastroesophageal varices. For this reason, understanding of PVTT must be improved.

This study reports a case of HCC with PVTT, and discusses the growth behavior of PVTT and how this affects the outcome of the disease.

\section{Case report}

A 43-year-old female with chronic hepatitis B was found to have advanced HCC with portal vein tumor thrombus (PVTT); this was detected in the right lobe using dynamic computed tomography (CT) on a routine check up at The 180th Hospital of The People's Liberation Army (Quanzhou, China). The patient had received prior treatment with interferon- $\alpha$ with $\mathrm{HBeAg}$ serological conversion.

Upon physical examination, the right subcostal liver could be palpated and no other significant findings were observed. The patient was hemodynamically stable. Laboratory data are shown in Table I. A marked increase in levels of tumor markers was observed ( $\alpha$-fetoprotein, 2,844.2 ng/ml; normal range, $0.0-10.0 \mathrm{ng} / \mathrm{ml})$. The patient's Child-Pugh score was 6 points (Class A) (8) and the Model for End-Stage Liver Disease score was 25 (9).

Dynamic CT imaging revealed an irregularly shaped tumor, $8.5 \times 7.0 \mathrm{~cm}$ in size. It was increased in size on early phase CT in the right lobe of the liver. A region of deficiency was observed 
A

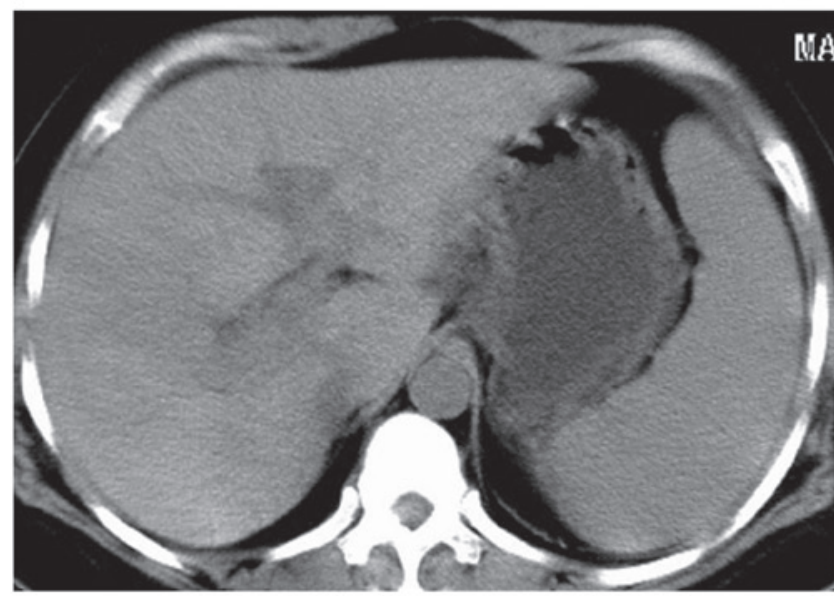

C

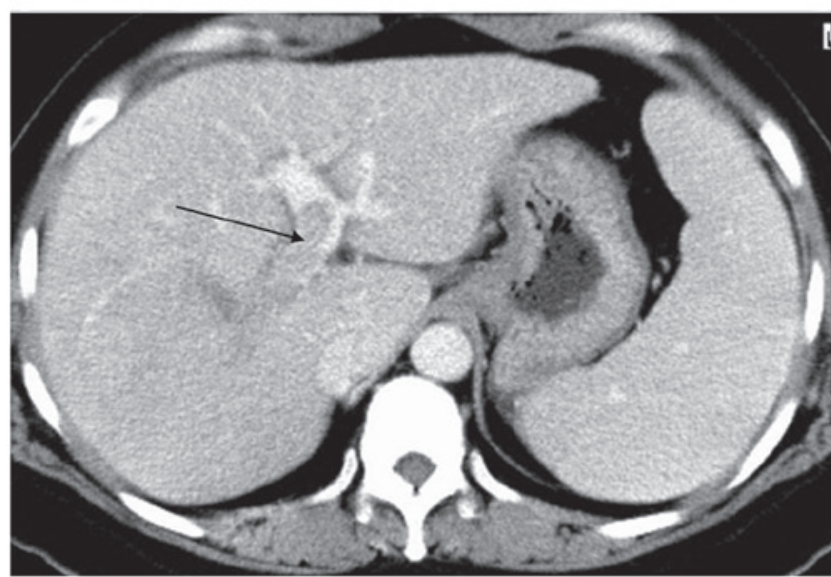

B

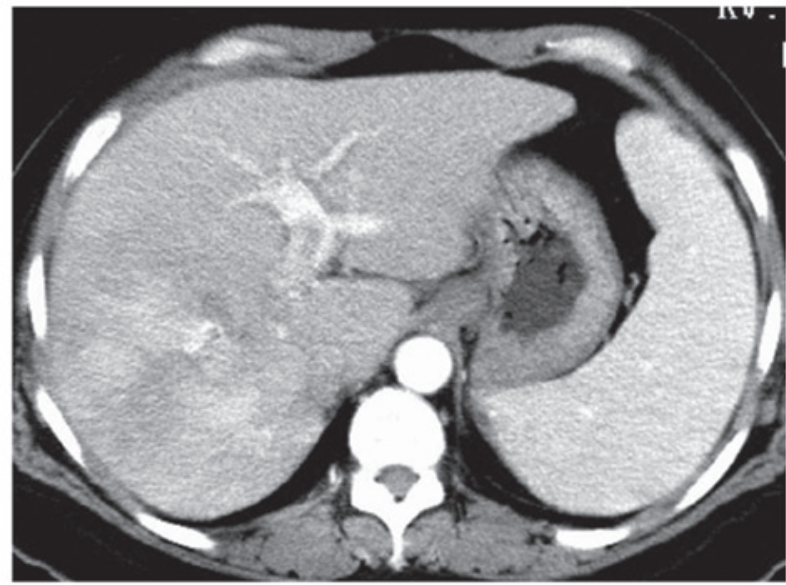

D

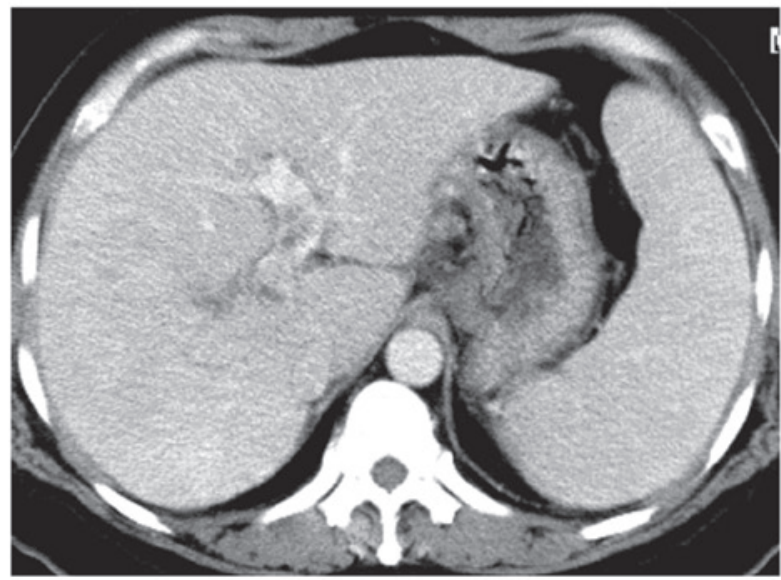

Figure 1. Dynamic computed tomography findings before treatment. (A) An irregularly shaped tumor of $8.5 \times 7.0 \mathrm{~cm}$ in size was identified in the right lobe of the liver. (B) The tumor increased in size during the early phase. (C and D) A region of deficiency was observed during the delayed phase. (B, C and D) The tumor invaded the left branch, right branch, and main portal vein and (C) was excised in the area indicated by the arrow.

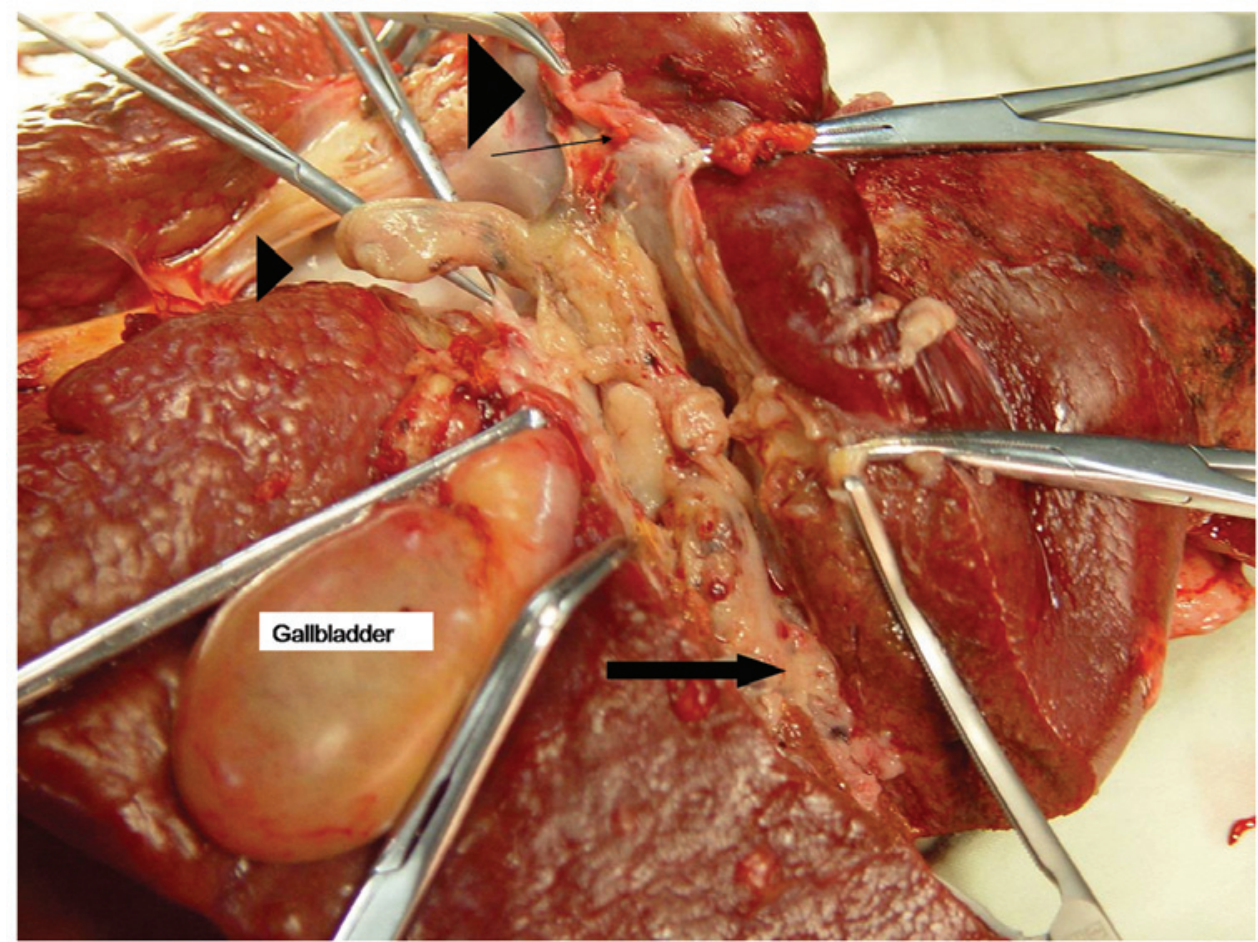

Figure 2. Anatomical examination of isolated liver with hepatocellular carcinoma with PVTT. The PVTT began at the primary tumor and grew from the right branch portal vein (large arrow) towards the main portal vein (large arrowhead) and the left branch of the portal vein (small arrowhead). A nutrient artery (small arrow) supplied the tumor thrombus. PVTT, portal vein tumor thrombosis. 
Table I. Laboratory data upon admission.

\begin{tabular}{|c|c|c|}
\hline Test & Result & Normal range \\
\hline \multicolumn{3}{|l|}{ Hematology } \\
\hline White blood cells per $\mu 1$ & 3,770 & 4.0-10.0 \\
\hline Red blood cells per $\mu 1$ & $418 \times 10^{4}$ & $409 \times 10^{4}-574 \times 10^{4}$ \\
\hline Hemoglobin, g/dl & 11.3 & 13.1-17.2 \\
\hline Platelets per $\mu 1$ & $9.4 \times 10^{4}$ & $10 \times 10^{4}-30 \times 10^{4}$ \\
\hline \multicolumn{3}{|l|}{ Coagulation } \\
\hline Prothrombin time, $\%$ & 90.0 & $70.0-130.0$ \\
\hline APTT, s & 32.6 & $20.0-40.0$ \\
\hline \multicolumn{3}{|l|}{ Virus markers } \\
\hline HBs antigen & Positive & Negative \\
\hline HCV antibody & Negative & Negative \\
\hline \multicolumn{3}{|l|}{ Tumor markers } \\
\hline AFP, ng/ml & $2,844.2$ & $0.0-10.0$ \\
\hline $\mathrm{CEA}, \mu \mathrm{g} / \mathrm{l}$ & 2.0 & $0.0-5.0$ \\
\hline \multicolumn{3}{|l|}{ Chemistry } \\
\hline Total protein, g/dl & 7.8 & $6.0-8.0$ \\
\hline Albumin, g/dl & 4.0 & $3.5-5.2$ \\
\hline Total bilirubin, $\mu \mathrm{mol} / 1$ & 18.4 & $1.7-20.0$ \\
\hline AST, IU/1 & 46.0 & $5.0-40.0$ \\
\hline ALT, IU/1 & 69.0 & $5.0-40.0$ \\
\hline ALP, IU/1 & 92.0 & $30.0-150.0$ \\
\hline$\gamma \mathrm{GTP}, \mathrm{IU} / 1$ & 163.0 & $7.0-50.0$ \\
\hline Choline esterase, IU/1 & $11,390.0$ & 4,300.0-13,200.0 \\
\hline LDH, IU/1 & 200.0 & 80.0-280.0 \\
\hline $\mathrm{Na}, \mathrm{mmol} / \mathrm{l}$ & 137.3 & $135.0-145.0$ \\
\hline $\mathrm{K}, \mathrm{mmol} / \mathrm{l}$ & 3.49 & $3.5-5.4$ \\
\hline $\mathrm{Cl}, \mathrm{mmol} / \mathrm{l}$ & 107.5 & $98.0-107.0$ \\
\hline BUN, mmol/1 & 4.5 & 1.8-8.9 \\
\hline $\mathrm{Cr}, \mu \mathrm{mol} / 1$ & 63.0 & $53.0-124.0$ \\
\hline
\end{tabular}

APTT, activated partial thromboplastin time; HBs, hepatitis B surface; HCV, hepatitis C virus; AFP, $\alpha$-fetoprotein; CEA, carcinoembryonic antigen; AST, aspartate aminotransferase; ALT, alanine aminotransferase; ALP, alkaline phosphatase; $\gamma$ GTP, $\gamma$-glutamyl transpeptidase; $\mathrm{LDH}$, lactate dehydrogenase; $\mathrm{Na}$, sodium; K, potassium; $\mathrm{Cl}$, chlorine; BUN, blood urea nitrogen; $\mathrm{Cr}$, creatinine.

on delayed phase CT imaging, in liver segment 8/6. The tumor had invaded the left and right branches, and main trunk of the portal vein (Fig. 1C, arrow). The PVTT began at the primary tumor, and extended towards the main portal vein, supplied by a nutrient artery. The PVTT tissue was extremely friable.

The patient subsequently underwent orthotopic liver transplantation. The gross surgical pathology of the isolated liver is shown in Fig. 2. Intraoperative histopathological examination also confirmed the diagnosis of HCC with PVTT. No complications were experienced, and the patient was discharged and subsequently treated with anti-viral and anti-rejection drugs in the outpatient clinic. The patient remained healthy during follow-up examinations. However, 8 years post-transplantation, metastatic lesions in the liver, lung and brain were detected by positron emission tomography-CT. The patient did not meet the recommended Milan Criteria for liver transplantation and therefore succumbed to intracranial hemorrhage at 51 years of age. This study was approved by the Institutional Review Board of The 180th Hospital of the People's Liberation Army. Written informed consent was obtained from the patient's family according to the principles expressed by the Declaration of Helsinki.

\section{Discussion}

HCC in combination with portal venous invasion is associated with poor outcome, and the extent of PVTT may affect the prognosis (10). In 2007, Shi et al (11) proposed a PVTT classification which was based on the extent of the tumor thrombus in the portal vein, as follows: Type I0, tumor thrombus formation found under microscopy; type I, tumor thrombi involving segmental branches of portal vein or above; type II, tumor thrombi involving right/left portal vein; type III, tumor thrombi involving the main portal vein trunk; and type IV, tumor 
thrombi involving the superior mesenteric vein (7). A number of studies have reported that patients with PVTT extending to the main trunk of the portal vein or beyond have poorer outcomes following surgery compared to those with PVTT confined to the first- or second-order portal branches (12). Therefore, the growth characteristics of PVTT may affect the outcomes in cases of advanced HCC. The presence of PVTT also limits the treatment options, as HCC treatment guidelines consider PVTT a contraindication for transplantation (13). Currently, treatment for HCC with PVTT includes surgery, radiotherapy, and transcatheter arterial embolization (TACE). Surgical resection is the optimal treatment modality for early-stage HCC patients, who account for only $5 \%$ of cases in Western countries and $\sim 40 \%$ of cases in Asia. The five-year survival rate of HCC patients after resection is $>50 \%$ (14). The five-year survival of early-stage HCC patients after liver transplantation is $>70 \%$, however, the lack of sufficient liver donation is the major limitation for liver transplantation (15). Kim et al (16) reported that radiotherapy for PVTT of HCC showed a complete response (CR) in 4 of 59 patients $(6.8 \%$ ), a partial response (PR) in 23 patients (39.0\%), no response in 28 patients $(47.5 \%)$ and progressive disease in four patients (6.8\%). TACE is considered for patients with inoperable HCC, who are not eligible for liver transplantation. Previous studies have reported that TACE treatment induced a CR or PR in $35 \%$ of patients, with $<2 \%$ of patients achieving a CR $(17,18)$. Lo et al $(19)$ showed that patients treated with TACE exhibited an increased two-year survival rate following treatment (41\%) when compared with patients receiving symptomatic treatment (27\%). TACE may improve survival in cases of intermediate-stage HCC with Child-Pugh class A in the absence of PVTT (19). Sorafenib has also been found to confer a survival benefit for patients with advanced HCC and PVTT, however, its safety and efficacy limit use (20).

In conclusion, in cases of HCC and PVTT where the growth characteristics and modality of PVTT include extension to the trunk of the portal vein or further, destruction of the primary tumor and PVTT must be considered. Patients with poor liver function or unresectable $\mathrm{HCC}$ who cannot receive surgery have a very poor prognosis, with a life expectancy of 2.7-4 months (6). In the present case, the PVTT was found to be supplied by a tumor vessel. This study supports use of the TAE methods and proposes that this approach may be a suitable for palliation of advanced HCC with PVTT in patients who cannot undergo a surgery.

\section{References}

1. Montalto G, Cervello M, Giannitrapani L, Dantona F, Terranova A and Castagnetta LA: Epidemiology, risk factors, and natural history of hepatocellular carcinoma. Ann NY Acad Sci 963: 13-20, 2002.
2. Yamanaka N, Okamoto E, Fujihara S, et al: Do the tumor cells for hepatocellular carcinomas dislodge into the portal venous stream during hepatic resection? Cancer 70: 2263-2267, 1992.

3. Nakashima T and Kojiro M: Hepatocellular Carcinoma. An Atlas of Its Pathology. 7th edition. Springer Verlag, Tokyo, 1987.

4. Wu MC, Chen H and Shen F: Surgical treatment of primary liver cancer: a report of 5524 patients. Chin J Surg 39: 25-28, 2001.

5. Zhang J, Gong F, Li L, et al: Diabetes mellitus and the neutrophil to lymphocyte ratio predict overall survival in non-viral hepatocellular carcinoma treated with transarterial chemoembolization. Oncol Lett 7: 1704-1710, 2014.

6. Llovet JM, Bustamante J, Castells A, et al: Natural history of untreated nonsurgical hepatocellular carcinoma: rationale for the design and evaluation of therapeutic trials. Hepatology 29: 62-67, 1999.

7. Lau WY, Lai EC and Yu SCH: Management Of Portal Vein Tumor Thrombus. In: Hepatocellular Carcinoma. Lau WY (ed). World Scientific Publishing, Singapore, pp739-760, 2008.

8. Pugh RNH, Murray-Lyon IM, Dawson JL, et al: Transection of the oesophagus for bleeding oesophageal varices. Br J Surg 60: 646-649, 1973

9. Kamath PS, Wiesner RH, Malinchoc M , et al: A model to predict survival in patients with end-stage liver disease. Hepatology 33 : 464-470, 2001

10. Shuqun C, Mengchao W, Han C, et al: Tumor thrombus types influence the prognosis of hepatocellular carcinoma with the tumor thrombi in the portal vein. Hepatogastroenterology 54: 499-502, 2007.

11. Shi J, Lai EC, Li N, et al: A new classification for hepatocellular carcinoma with portal vein tumor thrombus. J Hepatobiliary Pancreat Sci 18: 74-80, 2011

12. Chen XP, Qiu FZ, Wu ZD, et al: Effects of location and extension of portal vein tumor thrombus on long-term outcomes of surgical treatment for hepatocellular carcinoma. Ann Surg Oncol 13: 940-946, 2006

13. Bruix J and Sherman M; American Association for the Study of Liver Diseases: Management of hepatocellular carcinoma: an update. Hepatology 53: 1020-1022, 2011.

14. Arii S, Yamaoka Y, Futagawa S, et al: Results of surgical and nonsurgical treatment for small-sized hepatocellular carcinomas: A retrospective and nationwide survey in Japan. The Liver Cancer Study Group of Japan. Hepatology 32: 1224-1229, 2000.

15. Mazzaferro V, Regalia E, Doci R, et al: Liver transplantation for the treatment of small hepatocellular carcinomas in patients with cirrhosis. N Engl J Med 334: 693-699, 1996.

16. Kim DY, Park W, Lim DH, et al: Three-dimensional conformal radiotherapy for portal vein thrombosis of hepatocellular carcinoma. Cancer 103: 2419-2426, 2005.

17. Llovet JM and Bruix J: Systematic review of randomized trials for unresectable hepatocellular carcinoma: Chemoembolization improves survival. Hepatology 37: 429-442, 2003.

18. Bruix J, Sala M and Llovet JM: Chemoembolization for hepatocellular carcinoma. Gastroenterology 127 (5 Suppl 1): S179-S188, 2004.

19. Lo CM, Ngan H, Tso WK, et al: Randomized controlled trial of transarterial lipiodol chemoembolization for unresectable hepatocellular carcinoma. Hepatology 35: 1164-1171, 2002.

20. Federico A, Orditura M, Cotticelli G, et al: Safety and efficacy of sorafenib in patients with advanced hepatocellular carcinoma and Child-Pugh A or B cirrhosis. Oncol Lett 9: 1628-1632, 2015. 